\title{
FSL-1 Induces MMP-9 Production through TLR-2 and NF-KB /AP-1 Signaling Pathways in Monocytic THP-1 Cells
}

\author{
Rasheed Ahmad Puthiyaveetil Kochumon Shihab Sara Jasem \\ Kazem Behbehani
}

Immunology \& Innovative Cell therapy Unit, Dasman Diabetes Institute, Kuwait, Kuwait

\section{Key Words}

MMP-9 • TLR-2 $・$ MyD88 • p38・ERK

\begin{abstract}
Background: Matrix metalloproteinase-9 (MMP-9) is known to be implicated in the pathogenesis of many inflammatory disorders. FSL-1 (fibroblast-stimulating lipopeptide-1) induces cytokine production by monocytes/macrophages. However, it is unclear whether FSL-1 is also able to induce MMP-9 production. Herein, we determined whether FSL-1 could induce MMP-9 production, and if so, which signal transduction pathway(s) were involved. Methods: MMP-9 expression was assessed with real-time GPCR and ELISA. Signaling pathways were studied by using THP1-XBlue ${ }^{\text {TM }}$ cells, THP1-XBlue ${ }^{\text {TM }}$-defMyD cells, anti-TLR2 mAb and pharmacological inhibitors. Phospho and total proteins were determined by Western blotting. Results: FSL-1 induces MMP-9 expression $(P<0.001)$ at both mRNA and protein levels in human monocytic THP-1 cells. Elevated activity $(P<0.001)$ of NF-KB/AP-1 was also observed in FSL-1-treated THP-1 cells. FSL-1-induced MMP-9 secretion was markedly suppressed either by neutralizing anti-TLR-2 antibody or by inhibiting clathrin-dependent endocytosis. Furthermore, MyD88 $\%$ THP-1 cells did not express MMP-9 in response to FSL-1 treatment. By small interfering RNA-mediated knockdown, we also show that FSL-1-induced up-regulation of MMP-9 requires MyD88. Pre-treatment of THP-1 cells with inhibitors of JNK (SP600125), MEK/ERK (U0126; PD98056; XMD 8-92), p38 MAPK (SB203580) and NF-KB (BAY11-7085, Triptolide, Resveratrol) significantly suppressed $(P<0.05)$ MMP-9 gene expression and NF-KB/ AP-1 transcription factors activity. Conclusion: These findings provide the first evidence that FSL-1 induces TLR-2-dependent MMP-9 gene expression which requires the recruitment of MyD88 and leads to activation of MEK1/2 /ERK 1/2, MEK5/ERK5, JNK, p38 MAPK and NF-KB/ AP-1.

Copyright $@ 2014$ S. Karger AG, Basel

Dr. Rasheed Ahmad




\section{Introduction}

Matrix metalloproteinases (MMPs) are mostly secreted enzymes that belong to a family of zinc- and calcium-dependent proteolytic enzymes that are known to degrade the most components of the extracellular matrix (ECM) [1]. Based on their substrate specificity, MMPs are classified as collagenases, stromelysins, and gelatinases. MMP-2 and MMP9 are the gelatinases that have potential enzymatic activity against denatured collagens (gelatin), native type IV and V collagens and elastin. Type IV collagen and laminin are common components of the basement membrane [2]. MMP-9 is predominantly produced by monocytes/macrophages and is centrally involved in pathogenesis of cancer metastasis, obesity-induced inflammation, insulin resistance, asthma etc [3-6] Monocytes/macrophages abound in the sites of chronic inflammation such as areas of arthritis, atherosclerosis, and periodontal disease in which the connective tissue degradation by MMP-9 is considered to trigger the disease pathology [7]. To accomplish endothelial transmigration, monocytes transverse the sub-endothelial basement membrane and interstitial matrix that involves MMP-9-induced degradation of the basement membrane and ECM components. Inhibition of MMP-9 activation interrupted macrophage migration to the elicited site of inflammation [8]. MMP-9 is also reported to play an important role in obesity-mediated adipose tissue remodelling and obese individuals are known to have increased MMP-9 levels [9]. MMP-9 expression is typically absent or minimal in normal tissues and is greatly enhanced during inflammation and wound healing [10]. Its induction depends on activation of Toll-like receptors (TLRs) by different agonists such as pathogens associated molecular patterns (PAMPS) and other receptors activated by cytokines/chemokines [11].

TLRs are pattern-recognition receptors highly expressed on immune cells. All TLRs share an intracellular signaling motif with interleukin (IL)-1 receptor called toll-IL1-R (TIR) domain. Recognition of microbial invasion through TLRs activates signaling to recruit several adaptor proteins to the TIR domain. Except TLR3, all TLRs share a key adaptor molecule called myeloid differentiation (MyD)-88 factor which, once recruited to TIR domain, activates IL-1 receptor-associated kinases (IRAK) family members and tumor necrosis factor-alpha receptor-associated factor (TRAF)-6. Signaling for activation of the NF- $\mathrm{KB} / \mathrm{mitogen}$-activated protein kinase (MAPK) pathways involves these adaptor molecules. Expression of the key proinflammatory cytokines that regulate the immune and inflammatory responses involves critical transcription factors such as NF- $\mathrm{kB}$ and AP1 [12].

TLRs recognize the microbial lipoproteins which induce inflammation by causing NF$\kappa \mathrm{B}$ nuclear translocation resulting in cytokine production. MMP-9 is produced by various cells in response to the microbial infections [13-15]. Bacterial components are involved in the regulation of MMP-9. Wang et al. reported that peptidoglycan, a major wall component of gram positive bacteria, increases MMP-9 levels in the lung, liver and organ injury in the rat [16]. Stimulation of monocytes with LPS, a surface component of bacteria, induces a number of MMPs, including MMP-2 and MMP-9 [17]. FSL-1 (Fibroblast-stimulating lipopeptide-1; S-(2, 3 bispalmitoyloxypropyl)-CGDPKHSPKSF) is a synthesized diacylated lipopeptide mimicking a $44 \mathrm{kDa}$ lipoprotein of Mycoplasma salivarium known to activate the human gingival fibroblasts [18]. It induces expression of inflammatory cytokines such as IL-6, IL-8, monocyte chemotactic protein (MCP)-1 and tumor necrosis factor (TNF)- $\alpha$ by monocytes/ macrophages [19]. However, the role of FSL-1 in the regulation of MMP-9 in monocytic cells so far remains unclear. In addressing our study hypothesis that FSL-1 could activate the MMP-9 gene expression in monocytic cells, herein we report that FSL-1 induces MMP9 expression in monocytic THP-1 cells and it involves activation of the MAPK pathway and regulation through $\mathrm{NF}-\kappa \mathrm{B}$ and $\mathrm{AP}-1$ transcription factors. 


\section{Materials and Methods}

\section{Cell Culture}

Human monocytic leukemia cell line THP-1 was purchased from American Type Culture Collection (ATCC) and grown in RPMI-1640 culture medium (Gibco, Life Technologies, Grand Island, USA) supplemented with $10 \%$ fetal bovine serum (Gibco, Life Technologies, Grand Island, NY, USA), 2mM glutamine (Gibco, Invitrogen, Grand Island, NY, USA), 1mM sodium pyruvate, 10mM HEPES, 100ug/ml Normocin $50 \mathrm{U} / \mathrm{ml}$ penicillin and $50 \mu \mathrm{g} / \mathrm{ml}$ streptomycin (P/S; (Gibco, Invitrogen, Grand Island, NY, USA), and incubation at $37^{\circ} \mathrm{C}$ (with humidity) in $5 \% \mathrm{CO}_{2}$. THP-1-XBlue cells stably expressing a secreted embryonic alkaline phosphatase (SEAP) reporter inducible by NF- $\kappa \mathrm{B}$ and AP-1 were purchased from InvivoGen (InvivoGen, San Diego, CA, USA). THP-1-XBlue cells show similar response to FSL-1 as THP-1 cells. All the experiments were performed with THP-1-XBlue cells at a cell density of $1 \times 10^{6} / \mathrm{ml}$ in 12-well plates. THP-1-XBlue ${ }^{\mathrm{TM}}$-defMyD cells (cells deficient in MyD88 activity; MyD88-/- THP-1 cells) were also purchased from InvivoGen (InvivoGen, San Diego, CA, USA). THP-1-XBlue cells were cultured in complete RPMI medium with the addition of zeocin $(200 \mu \mathrm{g} / \mathrm{ml})$ (InvivoGen, San Diego, CA, USA) to select for cells expressing the SEAP -NF- $\mathrm{BB} / \mathrm{AP}-1$ reporter. THP-1-XBlue ${ }^{\mathrm{TM}}$-defMyD cells were cultured in complete RPMI medium with the addition of Zeocin (200ug/ $\mathrm{ml}$ ) and HygroGold (100ug/ml) (InvivoGen, San Diego, CA, USA) . Prior to stimulation, THP-1 cells were transferred into normal medium and plated in 12-well plates (Costar, Corning Incorporated, Corning, NY, USA) at $1 \times 10^{6}$ cells/well cell density unless indicated otherwise.

\section{Cell stimulation}

THP-1 cells were plated in 12-well plates (Costar, Corning Incorporated, Corning, NY, USA) at $1 \times 10^{6}$ cells/well concentration unless indicated otherwise. Cells were stimulated with FSL- 1 (a synthetic lipopeptide from Mycoplasma salivarium purchased from Invivogen; InvivoGen, San Diego, CA, USA) (50ng/ $\mathrm{ml}$ ) or TNF- $\alpha$ (25nglml) for $24 \mathrm{hr}$ at $37^{\circ} \mathrm{C}$. Cells were harvested for RNA isolation and conditioned media were collected for measuring MMP-9 secretion levels and SEAP activity. Conditioned media were collected and stored at $-80^{\circ} \mathrm{C}$.

\section{Cell Transfection}

THP-1 cells were washed and resuspended in fresh medium without antibiotics and transfected separately with psiRNA-hMyD88 (InvivoGen, CA, USA), psiRNA-LucGL3 (InvivoGen, CA, USA) and pmaxGFP (provided in Amaxa Noclecfector Kit V for THP-1, Lonza). 0.5ug of each psiRNA was used for the trasfection of $1 \times 10^{6}$ THP- 1 cells. All transfection experiments were performed with Amaxa Cell Line Nucleofector Kit V for THP-1 (Lonza, Germany) by using Amaxa Electroporation System (Amaxa Inc, Germany)) according to the manufacturer's protocol. Tranfection efficiency was analysed by fluorescence microscopy. 40-54\% THP-1 cells were trasfected with the plasmid. After $48 \mathrm{hr}$ transfection, cells were treated with FSL-1 (50 ng/ $\mathrm{ml}$ ) for $24 \mathrm{hrs}$.

\section{Measurement of $N F-\kappa B / A P-1$ activity}

THP-1 XBlue cells (InvivoGen, San Diego, CA) are THP-1 cells stably transfected with a reporter construct, expressing a secreted embryonic alkaline phosphatase (SEAP) gene under the control of a promoter inducible by the transcription factors NF- $\kappa$ B and AP-1. Upon stimulation, NF- $\kappa \mathrm{B}$ and AP-1 are activated and subsequently the secretion of SEAP is promoted. THP-1 XBlue cells were stimulated with FSL-1 (50ng/ml) or TNF- $\alpha\left(25 \mathrm{ng} / \mathrm{ml}\right.$; positive control) for $24 \mathrm{hr}$ at $37^{\circ} \mathrm{C}$. Levels of SEAP were detected in the conditioned media after $4 \mathrm{hr}$ incubation of supernatants with Quanti-Blue medium (InvivoGen, San Diego, CA, USA) at $650 \mathrm{~nm}$ wave length by ELISA reader.

\section{Real Time Quantitative RT-PCR}

Total RNA was extracted using RNeasy Mini Kit (Qiagen, Valencia. CA, USA). The cDNA was synthesized using $1 \mu \mathrm{g}$ of total RNA using high capacity cDNA reverse transcription kit (Applied Biosystems, Foster city, CA, USA). 50ng cDNA was used in each real-time PCR reaction. For real-time polymerase chain reaction (PCR), complementary DNA was amplified with Inventoried TaqMan Gene Expression Assay products (MMP9: Hs00234579_m1; GAPDH: Hs03929097_g1) containing two gene-specific primers and one TaqMan MGB probe (6-FAM dye-labeled) using a TaqMan® Gene Expression Master Mix (Applied Biosystems, Foster city, 
CA, USA) in a 7500 Fast Real-Time PCR System (Applied Biosystems, Foster City, CA, USA). The mRNA levels were normalized against GAPDH mRNA and the amounts of MMP-9 mRNA relative to control were calculated with $\Delta \Delta$ Ct-method [20]. Relative mRNA expression was expressed as fold expression over average of control gene expression. The expression level in control treatment was assumed to be 1 . Values are presented as mean \pm SEM. Results were analyzed statistically; $P<0.05$ was considered significant.

\section{Secreted MMP-9 quantification}

MMP-9 secreted protein in supernatants of THP-1 cells stimulated with FSL-1 or TNF- $\alpha$ was quantified using sandwich ELISA following the manufacturer's instructions ((R\&D systems, Minneapolis, USA).

\section{Western blotting}

THP-1 cells were harvested and incubated for $30 \mathrm{~min}$ with lysis buffer (Tris $62.5 \mathrm{mM}$ (pH 7.5), 1\% Triton X-100,10\% glycerol). The lysates were then centrifuged at $14000 \mathrm{rpm}$ for $10 \mathrm{~min}$ and the supernatants were collected. Protein concentration in the lysates was measured by Quickstart Bradford Dye Reagent , 1x Protein Assay kit (Bio-Rad Laboratories, Inc, CA). Protein (20 $\mu \mathrm{g})$ samples were mixed with sample loading buffer, heated for $5 \mathrm{~min}$ at $95^{\circ} \mathrm{C}$ and resolved by $12 \%$ SDS-PAGE. Cellular proteins were transferred to Immuno-Blot PVDF membrane (Bio-Rad Laboratories, USA) by electro blotting. The membranes were then blocked with 5\% non-fat milk in PBS for $1 \mathrm{~h}$, followed by incubation with primary antibodies against p-MEK1/2, pERK1/2, p-JNK, p-p38, p-c-jun, p-IKK $\alpha / \beta, p-I K B, p-N F-K B$ and beta actin in 1:1000 dilution at $4^{\circ} \mathrm{C}$ overnight. All the primary antibodies were purchased from Cell Signaling (Cell Signaling Technology, Inc). The blots were then washed four times with TBS and incubated for $2 \mathrm{~h}$ with HRP-conjugated secondary antibody (Promega, Madison, WI, USA). Immunoreactive bands were developed using an Amersham ECL Plus Western Blotting Detection System (GE Health Care, Buckinghamshire, UK) and visualized by Molecular Imager $\AA_{\text {VersaDoc }}^{\mathrm{TM}}$ MP Imaging Systems (Bio-Rad Laboratories, Hercules, CA, USA).

\section{Statistical analysis}

Statsitical analysis was performed using GraphPad Prism software (La Jolla, CA, USA). Data are shown as mean \pm standard deviation values, unless otherwise indicated. Unpaired Student t-test was used to compare means between groups. For all analyses, $P$ value $<0.05$ was considered significant.

\section{Results}

FSL-1 induces MMP-9 gene expression in THP-1 cells

In previous studies, it has been shown that FSL-1 activates different inflammatory mediators [21]. However, since MMP-9 induction by FSL-1 in THP-1 cells has yet not been studied, we are looking at the impact of FSL-1 on the regulation of MMP-9 gene expression. To investigate whether FSL-1 can induce gene expression of MMP-9, we treated THP-1 cells with commercially available mycoplasma-derived peptide FSL-1 (50ng/ml) for 24 hours. Cells and conditioned media were harvested. MMP-9 gene expression in cells was determined by real-time RT-PCR using total cellular mRNA. Conditioned medium was used to measure the concentrations of MMP-9 secreted protein by ELISA. Our data show (Fig. 1A) that MMP-9 mRNA expression levels were significantly higher (346 Fold; $P=0.001$ ) in FSL1-treated THP-1 cells than those of controls (cells treated with vehicle only). Concordantly, MMP-9 soluble protein levels (Fig. 1B) were significantly higher in THP-1 cell supernatants after stimulation with FSL-1 (2424 $\pm 10 \mathrm{pg} / \mathrm{ml} ; P<0.0001)$.

As the promoter of both the human and murine MMP-9 genes has NF- $\mathrm{B} / \mathrm{AP}-1$ sites and It has been observed the activation NF- $\kappa \mathrm{B} / \mathrm{AP}-1$ which is playing a role in the induction of MMP-9 in monocytic cells challenged with different components of pathogens and cytokines[22, 23]. This led us to investigate whether FSL-1 induced enhanced levels of NF$\kappa \mathrm{B} / \mathrm{AP} 1$ activity in THP-1 cells. We confirmed the dependence of FSL-1-induced MMP-9 production on NF- $\mathrm{B}$ /AP1 activation using THP-1 cells expressing a reporter driven by NF$\kappa \mathrm{B}$ and AP-1 response elements. Conditioned media obtained from FSL-1-activated THP-1 cells were analyzed for NF- $\kappa \mathrm{B} / \mathrm{AP}-1$ activity. As expected, NF- $\kappa \mathrm{B} / \mathrm{AP}-1$ activity was strongly 
Fig. 1. Effect of FSL-1 on MMP-9 expression in THP1 cells. THP-1 cells were cultured in 12-well plates at a concentration of $1 \times 10^{6}$ cells/well. Cells were treated with FSL-1 (50ng/ml), TNF- $\alpha$ (25 ng/ml; positive control) and vehicle $\left(\mathrm{H}_{2} \mathrm{O}\right.$; $2 \mathrm{ul} / \mathrm{ml}$ ). At $24 \mathrm{hr}$, cells and culture supernatants were harvested. Total cellular RNA was isolated and MMP-9 gene


expression was determined by real-time quantitative RT-PCR. Relative mRNA expression was expressed as fold expression over average of gene expression in vehicle-treated cells. The average gene expression level in vehicle-treated cells was assumed to be 1 (A). Cell culture supernatants were assayed for (B) secreted MMP-9 protein as determined by ELISA and (C) SEAP reporter activity (degree of NF- $\kappa \mathrm{B} / \mathrm{AP}-1$ activation). The results obtained from three independent experiments are shown. The data are presented as mean \pm SE. An asterisk $(*)$ represents $P$-value of $<0.05$.

Fig. 2. TLR-2 neutralizing antibody blocks the FSL1-induced MMP-9 upregulation. THP-1 cells were treated separately with $1 \mu \mathrm{g} / \mathrm{mL}$ of anti-huTLR-2-IgA (neutralizing mAb; Nab) or isotype-matched control antibody (IgA) for 30 minutes. Non-treated cells as well as antibody-treated cells were exposed to FSL-1 $(50 \mathrm{ng} / \mathrm{ml})$. Cells and cell culture supernatants were collected at $24 \mathrm{hr}$ after FSL-1 treatment. Cells were used for isolation of total RNA to assess the MMP9 gene expression by real-time RT-PCR (A). Cell supernatants were assayed for secretion of (B) MMP9 protein and (C) SEAP reporter activity (degree of AP-1/NF- $\kappa$ B activation). Data are shown from three independent experiments. The results are presented as mean \pm SE. An asterisk $(*)$ represents $P$-value of $<0.05$.



induced $(\mathrm{P}<0.001)$ in conditioned media from FSL-1-treated cells (Fig. 1C) and this correlated with MMP-9 secretion. These data suggest that FSL-1 treatment causes the release of MMP-9 from monocytic cells through the activation of NF- $\kappa \mathrm{B}$ and AP-1 transcription factors.

FSL-1 induces the TLR-2-dependent regulation of MMP-9

FSL-1 can induce target cells either through stimulating a specific surface receptor or through endocytic uptake. We used TLR-2 receptor blocking antibody and isotype control 
Fig. 3. Effect of endocytosis inhibitor on TLR2 mediated MMP-9 induction. THP-1 cells were pretreated with chlorpromazine (an inhibitor of clathrin dependent endocytosis; $10 \mathrm{uM}$ ) and Methyl- $\beta$-cyclodextrin (an inhibitor clathrin independent endocytosis; $2 \mathrm{mM}$ ) for $1 \mathrm{hr}$ and then treated with FSL-1 for $24 \mathrm{hr}$. Cells and supernatants were collected. Cells were used for the isolation of total RNA to assess the MMP-9 gene expression by real-time RT-PCR (A). Cell culture supernatants were assayed for (B) secreted MMP-9 protein as determined by ELISA and (C) SEAP reporter activity (degree of NF- $\kappa \mathrm{B} / \mathrm{AP}-1$ activation). The results obtained from three independent experiments are shown. The data are presented as mean \pm SE. An asterisk $\left({ }^{*}\right)$ represents $P$-value of $<0.05$.

Fig. 4. MMP-9 gene expression is MyD88-dependent. THP-1-XBlue ${ }^{\mathrm{TM}}$ defMyD cells (Cells deficient in MyD88 activity) were treated with FSL-1 (50ng/ml), TNF- $\alpha$ (25ng/ $\mathrm{ml}$ ) and vehicle (water; 2ul) for $24 \mathrm{hr}$. Cells and supernatants were collected. Cells were used for the isolation of total RNA to assess the MMP-9 gene expression by real-time

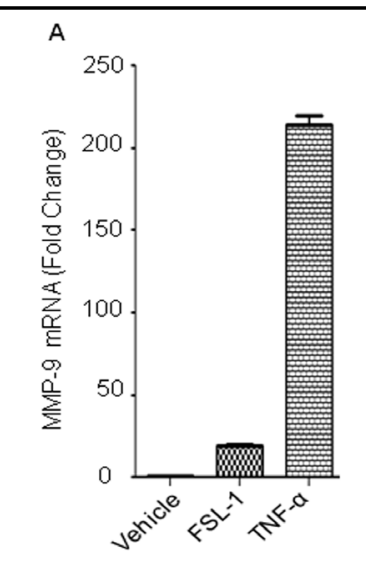

c





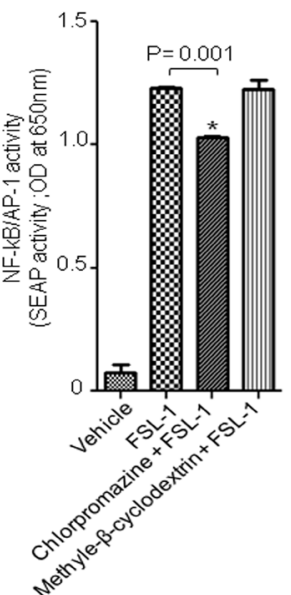

RT-PCR (A). Secreted levels of MMP-9 protein were determined in supernatants by ELISA (B). SEAP reporter activity (degree of AP-1/NF- $\mathrm{KB}$ activation) was also determined in cell supernatants (C). Data are shown as mean \pm SE. An asterisk $\left({ }^{*}\right)$ represents $P$-value of $<0.05$.

antibody to treat cells before stimulation with FSL-1. Anti-TLR-2 antibody significantly decreased the FSL-1-induced MMP-9 gene expression in THP-1 cells (Fig. 2A; $P=0.02$ ) as well as reduced secreted MMP-9 protein levels in the conditioned media (Fig. $2 \mathrm{~B} ; P=0.0008$ ). However, TNF- $\alpha$-induced MMP-9 gene expression did not depend on TLR- 2 signaling which was used as a positive control. This finding suggests that FSL-1 induces MMP-9 gene expression by signaling through TLR-2 receptor. Furthermore, pre-treatment of cells with anti-TLR-2 antibody resulted in decreased activity of NF- $\kappa$ B and AP-1 (Fig. 2C; P=0.023).

Studies showed an important role for the internalization of TLR-2 expressed at the plasma membrane by a process dependent on clathrin and triggers specific signals to activate some genes [24-26]. To assess the role of TLR-2 internalization in the induction of MMP-9, THP1 cells were treated with chlorpromazine (an inhibitor of clathrin dependent endocytosis) (Sigma-Aldrich, MO, USA) and methyl- $\beta$-cyclodextrin (an inhibitor of clathrin independent endocytosis) (Sigma-Aldrich, MO, USA). Treatment of the cells with chlorpromazine resulted in inhibition of MMP-9 induction by FSL-1 (Fig 3A and B). However, methyl- $\beta$-cyclodextrin did not show any impact on the MMP-9 induction (Fig 3A and B). Consistent with MMP- 
Fig. 5. MyD88 siRNA knockdown affects FSL-1 induced MMP-9. THP-1 cells were transfected with psiRNAhMyD88 (a silencing plasmid expressing a siRNA targeting the human MyD88 gene or psiRNALucGL3 (a control psiRNA plasmid) by Amaxa electroporation system. MyD88 deficient THP1 cells or wild type THP1 cells were treated with FSL-1 (50ng/ml) for $24 \mathrm{hr}$. Cells and supernatants were collected. Cells were used for the isolation of total RNA to assess the MMP-9 gene

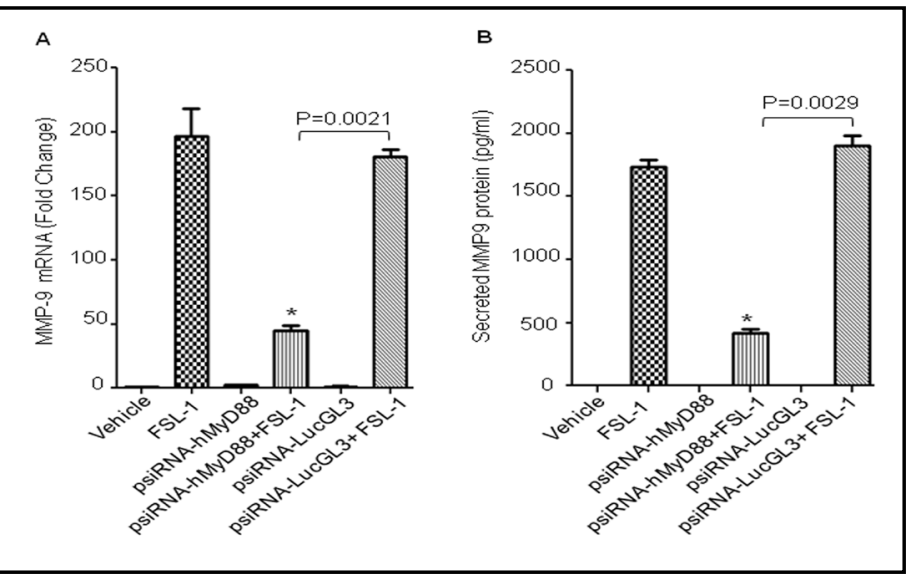
expression by real-time RT-PCR (A). Secreted levels of MMP-9 protein were determined in supernatants by ELISA (B). Data are shown as mean \pm SE. An asterisk ( $*$ ) represents $P$-value of $<0.05$.

9 gene expression results, FSL-1 induced NF- $\kappa \mathrm{B} / \mathrm{AP}-1$ activity was significantly reduced $(\mathrm{P}<0.001)$ in conditioned media obtained from the THP-1 cells treated with chlorpromazine (Fig. 3C). These results indicate that TLR-2 internalization facilitates NF-kB/AP-1 activation and resulting MMP-9 expression in response to FSL-1.

\section{MMP-9 induction by FSL-1 involves recruitment of MyD88 adaptor}

It is well known that MyD88 is a key adaptor molecule involved in the TLR-mediated signaling and triggering of downstream cascades which regulate the inflammatory mediators [27]. To determine whether MyD88 could be involved in the induction of MMP-9, THP-1$\mathrm{XBlue}^{\mathrm{TM}}$-defMyD cells (cells deficient in MyD88 activity; MyD88-/- THP-1 cells) were treated with FSL-1 or TNF- $\alpha$. Following FSL-1 treatment, MMP-9 expression was significantly reduced in MyD88-/- cells at both mRNA and protein levels (Fig. 4A and B). On the other hand, TNF- $\alpha$ induction of MMP-9 was not affected in MyD88-/- cells as it activates MMP-9 gene expression via MyD88-independent pathway. Furthermore, by using siRNA approach we confirmed the role of MyD88 in MMP-9 induction. These MMP-9-inducing effects of FSL1 were decreased in THP-1 cells transfected with MyD88-specific psiRNA (Fig 5A and B). These results demonstrate that FSL-1-induced MMP-9 gene expression requires expression of MyD88 adaptor protein. Similarly, MyD88 deficiency also led to significantly reduced activation of NF- $\kappa B / A P-1$ following FSL-1 treatment (Fig. 4C). These data suggest that FSL1-induced up-regulation of MMP-9 gene expression in monocytic cells involves activation of NF- $\kappa \mathrm{B} / \mathrm{AP}-1$ transcription factors through the MyD88-dependent signaling cascade.

\section{Signaling pathways mediating FSL-1 induced MMP-9 gene expression}

It was previously reported that MAP kinase signaling (ERK, JNK, p38) mediated MMP-9 release; we next asked whether these molecules also regulated the FSL-1-stimulated MMP-9 expression. Stimulation of THP-1 cells by FSL-1 increased MEK/ERK phosphorylation (Fig. 6A). Treatment with MEK inhibitors, such as U0126 or PD98059, resulted in inhibition of ERK1/2 phosphorylation (Fig. 6B), indicating that FSL-1 induced the activation of MEK1/2// ERK1/2 pathways in THP-1 cells. We found that JNK and p38 MAPK were also activated as indicated by the increased phosphorylation of JNK and p38 after FSL-1 treatment (Fig. 6A). Pre-treatment with JNK inhibitor (SP600125) resulted in inhibition of c-Jun phosphorylation (Fig. 6C). The expression of MMP-9 mRNA was blocked (Fig. 7A; $P<0.001$ ) by treatment with inhibitors of either p38-MAPK/JNK (SB203580/SP600125: InvivoGen, CA, USA) or MEK/ ERK (PD98059, U0126: InvivoGen, CA; XMD 8-92: Tocris Bioscience, UK). Consistent with qRT-PCR results, MMP-9 levels in culture supernatants of THP-1 cells were significantly 


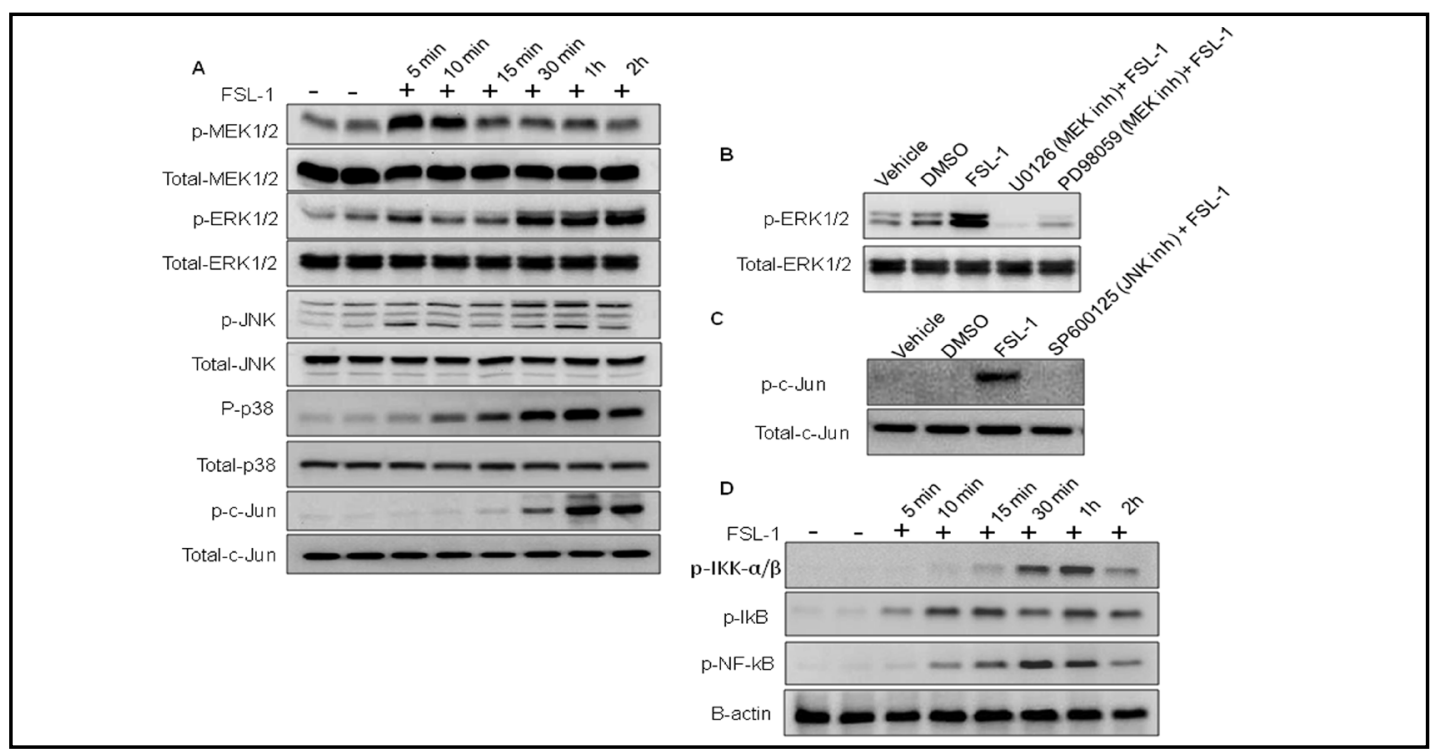

Fig. 6. FSL-1 activates the MAP kinase/NF- $\kappa B$ signaling pathway. THP-1 cells were treated with FSL-1 for different time points and cell lysates were prepared as described in methods. Samples were run on denaturing gels. Phosphorylated MEK1/2, ERK1/2, JNK, p-38 and c-Jun are depicted in the upper panels and total respective proteins are shown in the lower panels. As shown, FSL-1 treatment increases the phosphorylation of MEK1/2, ERK1/2, JNK, p38 and c-Jun in a time-dependent manner (A). Cells were pretreated with MEK-ERK inhibitors (U0126: 10uM; PD98059: 10uM) or JNK inhibitor (SP600125: 20uM) for $1 \mathrm{hr}$ and then treated with FSL- 1 for $1 \mathrm{hr}$. Phosphorylated ERK1/2 and c-Jun are depicted in the upper panels and total respective proteins are shown in the lower panels (B and C). Phosphorylated IKK $\alpha / \beta, \mathrm{IkB}$, and NF- $\mathrm{B}$ are depicted in the upper panel and beta actin is shown in the bottom panel (D).

diminished $(P<0.001)$ after treatment with inhibitors of either MAPKs or MEK/ERK (Fig. $7 \mathrm{~B})$. Given that the promoter contains the NF- $\kappa \mathrm{B}$ binding site, the loss of NF- $\kappa \mathrm{B}$ activation is expected to result in the reduced MMP-9 gene expression [28, 29]. Our results show that

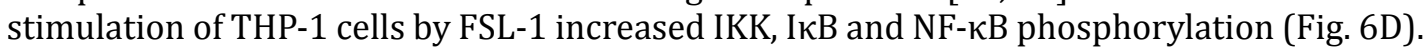
The use of NF- $\kappa$ B inhibitors (BAY 11-7805, Triptolide and Resveratrol: InvivoGen, CA, USA), which significantly reduced $(P<0.0001)$ the MMP-9 gene expression (Fig. $8 \mathrm{~A}$ and $8 \mathrm{~B})$ with diminished $(P<0.01)$ NF- $\kappa$ B activation (Fig. 8C).

\section{Discussion}

FSL-1 is known to activate different inflammatory mediators [14]; however, induction of MMP-9 in monocytic THP-1 cells has not been studied as yet. In the present study, we have analyzed the impact of FSL-1 on MMP-9 gene expression in monocytic cells. Our data show that FSL-1 induces an increase in the MMP-9 expression in THP-1 cells and this induction is dependent on activation of the MAPK/NF- $\mathrm{KB} / \mathrm{AP}-1$ pathway. Matrix metalloproteinases are implicated in the degradation of extracellular matrix components and hence in the mechanisms regulating cellular migration and tissue modeling [30, 31]. Highly-conserved microbial components, such as lipopolysaccharide (LPS), lipopeptides (LPs), lipoteichoic acids (LTAs), and peptidoglycan (PGN) serve as markers for the recognition of pathogens by TLRs in mammals and induce the production of cytokines and other inflammatory mediators [32-37]. Herein, we showed that blockade the cell surface expression of the TLR-2 significantly suppressed the induction of MMP-9, indicating that MMP-9 gene expression by FSL-1 in THP-1 cells was TLR-2-dependent. Furthermore, FSL-1 induced up-regulation of MMP-9 is significantly inhibited by blocking clathrin- dependent endocytosis. Interactions 


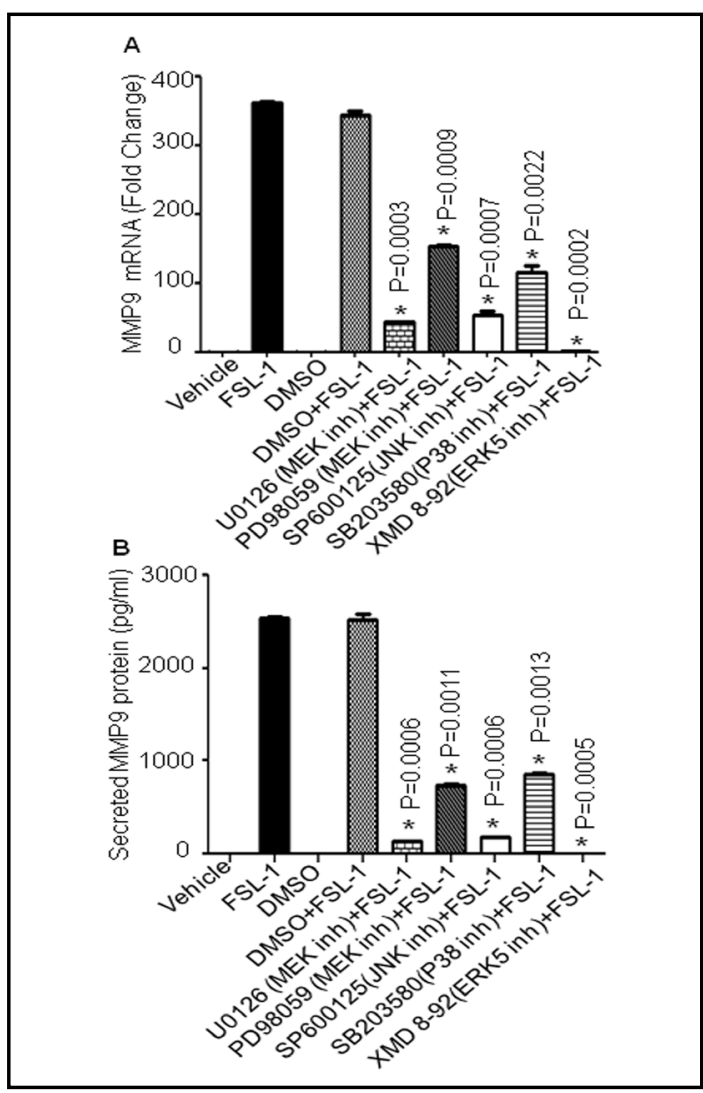

Fig. 7. Effect of MAPK pathway inhibitors on MMP-9 induction. THP-1 cells were pretreated with MEKERK inhibitors (U0126: 10uM; PD98059: 10uM) or JNK inhibitor (SP600125: 20uM) or p38 inhibitor (SB203580: 10uM) or MEK5/ERK5 inhibitor (XMD 8-92: $1 \mathrm{uM}$ ) or for $1 \mathrm{hr}$ and then treated with FSL-1 for $24 \mathrm{hr}$. Cells and supernatants were collected. Cells were used for the isolation of total RNA to assess the MMP-9 gene expression by real-time RT-PCR (A). Secreted levels of MMP-9 protein were determined in supernatants by ELISA (B). The results obtained from three independent experiments are shown. The data are presented as mean \pm SE. An asterisk $\left({ }^{*}\right)$ represents $P$-value of $<0.05$.



Fig. 8. NF- $\kappa B$ inhibitors suppress the FSL-1-induced MMP-9 gene expression. THP-1 cells were pretreated with NF- $\kappa$ B inhibitors (BAY 11-7085, $10 \mu \mathrm{M}$; Triptolide, $10 \mu \mathrm{M}$ or Resveratrol, 15uM) for $1 \mathrm{hr}$ and then treated with FSL-1 (50ng/ml) for $24 \mathrm{hr}$. Cells and supernatants were collected. Cells were used for the isolation of total RNA to assess the MMP-9 gene expression by real-time RT-PCR (A). Secreted levels of MMP-9 protein were determined in supernatants by ELISA (B). SEAP reporter activity (degree of AP-1/NF- $\kappa$ B activation) was also determined in cell supernatants (C). Data are shown as mean \pm SE. An asterisk $\left(^{*}\right)$ represents $P$-value of $<0.05$.

of different bacterial components with TLR- 2 and TLR- 4 are known to dictate the outcome of immune response and bacterial pathogenesis. TLR-4 mediates LPS signaling while TLR2 is the signaling receptor for molecules such as LP (FSL-1), LTA, and PGN [38, 39]. TLR2 is also implicated in the recognition of Gram-positive bacterial components, bacterial lipoproteins, and zymosan [40]. Accordingly, TLR-2-deficient mice displayed impaired cytokine production in response to PGN and Mycoplasma lipopeptide [36]. TLR-mediated signaling involves an important adapter protein MyD88 which is a key adaptor molecule involved in TLR-mediated signaling and triggering of downstream cascades regulating the inflammatory mediators [41]. Using MyD88-/- THP-1 monocytic cell line model or MyD88 siRNA, we show here that FSL-1-induced upregulation of the MMP-9 gene expression in these cells involved a MyD88-dependent signaling pathway. These results further confirm the involvement of TLRs as sensors of FSL-1 which initiates the MyD88-dependent signaling 
cascade that ultimately leads to the activation of MMP-9 gene expression through the transcription factors NF- $\kappa B$ and AP-1. Targeted disruption of the MyD88 gene resulted in the abrogation of NF- $\kappa B$ and AP-1 activities in response to FSL-1 stimulation. Studies using MyD88-deficient mouse model have revealed that this factor plays a critical role in the production of proinflammatory cytokines in response to TLR ligands [42]. It has been found that MyD88-deficient THP-1 cells display a defective response to many microbial components [43]. Our findings are corroborated by the previous study showing that cellular responses to bacterial cell wall components involved MyD88-dependent signaling cascades [44-46]. Notably, MyD88 is essential for cellular responses to IL-1, IL-18, and many bacterial cell wall components such as LPS, PGN, and LPs [41, 44, 47].

We further investigated the signaling pathways involved in the induction of MMP-9 by FSL-1 in THP-1 cells. It was previously shown that the engagement of TLRs with their agonists induced the activation of several signaling pathways, such as IKK/NF- $\kappa B, M A P K$ (p38, p42, and p44) and JNK in different cell types [48]. NF- $\mathrm{B}$ /AP1 activation has been observed after binding of bacterial components to their target cells [49-51]. Our data show that the signaling pathways triggered in response to FSL-1-induced MMP-9 production include MEK1/2/ERK1/2, MEK5/ERK5, p38 MAPK, JNK, c-Jun and NF- $\kappa$ B/AP-1. Activation of the ERK and p38 was reported to occur in response to $M$. fermentans membrane fractions [52]. Wilhelmsen et al. reported that NF- $\kappa B$ and the MAPK family members- p38-MAPK, JNK, and ERK5 are all required for the TLR2-induced up-regulation of inflammatory proteins [53]. NF- $\kappa \mathrm{B}$ and AP-1 triggering by FSL-1 also involved MAPK pathways. In agreement with previously published data [54-59] indicating that the p38 MAPK was required for the activation of several transcription factors, including c-Fos and c-Jun, we hereby show that MAPK pathway activation is required for AP-1 induction in response to FSL-1 [53]. Interestingly, MAPK pathway is also required for NF- $\kappa B$ activation in FSL-1-stimulated THP1 cells. At present, it is unclear whether MAPKs affects NF- $\kappa B$ function directly or indirectly. As our data show, pre-treatment of THP- 1 cells with the inhibitors of MEK1/2-ERK1/2, MEK5/ERK5, JNK, p38 and NF- $\kappa$ B greatly attenuated the MMP-9 induction. It is known that MMP-9 expression is regulated by p38, JNK and NF-kB [60]. Activation of AP-1 is consistent with the strong activation of JNK and ERK1/2 by bacterial cell wall components [61] and both JNK and ERK are involved in FSL-1 induced MMP-9 regulation as we observed. MEK/ ERK pathway has been clearly indicated to contribute to cell signaling resulting in cytokine production in response to FSL-1-mediated NF- $\kappa$ B or AP-1 transactivation [53]. Following FSL-1 treatment, phosphorylation of the JNK and c-Jun suggests that the JNK pathway is crucial for transducing these signals. Inhibition of the FSL-1 induction of MMP-9 by JNK inhibitors corroborates essential requirements of JNK pathway. The reported effects of JNK inhibitors on the activity of NF- $\kappa$ B further establish a necessary role for JNK-c-Jun pathway [62]. Altogether our results support the hypothesis that JNK, MEK1/2-ERK1/2, MEK5/ ERK5, p38 MAPK and -NF- $\mathrm{KB} / \mathrm{AP}-1$ are important components of the FSL-1-mediated MMP9 production in THP-1 monocytic cells.

In summary, we show here for the first time that FSL-1 can induce the production of MMP-9 inmonocytic THP-1 cells and that this MMP-9 gene induction is dependent on signaling through the JNK, MEK1/2-ERK1/2, MEK5/ERK5, p38 MAPK and NF- $\kappa B$ / AP-1 trans-activation. The excessive MMP activity that is observed following infection is expected to cause tissue damage. MMP-9 mediated regulation of matrix environment results a large neutrophil influx at the sites of inflammation and plays a more destructive than protective role in host defense against infection. Many pathological conditions caused by bacterial components are characterized by over-expression of MMP-9 and suggesting that tight regulation of MMP-9 gene is important for normal homeostasis. Understanding the molecular mechanisms controlling MMP-9 gene expression might identify new therapeutic targets. Thus, we suggest that blocking of the target molecule(s) in the signaling cascades activated by MMP-9 in response to microbial pathogens may help alleviate the aggressiveness of infections that may cause severe inflammatory disorders in individuals, especially those with immune deficiencies. 


\section{Abbreviations}

AP-1 (Activating protein-1); ATCC (American Type Culture Collection); ECM (Extracellular matrix ); ELISA (Enzyme-linked immunosorbent assay); ERK (Extracellular signal-regulated kinase); FBS (Fetal bovine serum); FSL-1 (Fibroblast-stimulating lipopeptide-1); GAPDH (Glyceraldehyde-3-phosphate dehydrogenase); IkB (NF-kB inhibitor); IKK (Inhibitor kappa B kinase); IRAK (IL-1 receptor-associated kinases family); JNK (c-Jun NH2-terminal kinase); LPs (Lipopeptides); LPS (Lipopolysaccharide); LTAs (Lipoteichoic acid); MAPK (Mitogen-activated protein kinase); MCP-1 (Monocyte chemotactic protein ); MEK (Mitogen-activated protein kinase/extracellular signal-regulated kinase); MMP-9 (Matrix metalloproteinase-9); MyD88 (Myeloid differentiation factor 88); NF-кB (Nuclear factor-kappaB); p38 MAPK (p38 mitogen-activated protein kinase); PAMPS (Pathogens associated molecular patterns); PCR (Polymerase chain reaction); PGN (Peptidoglycan); SEAP (Secreted embryonic alkaline phosphatase); THP1 (A human monocytic cell line); TIR (Interleukin (IL)-1 receptor called toll-IL1-R); TLRs (Toll-like receptors); TNF- $\alpha$ (Tumor necrosis factor-alpha); TRAF-6 (Tumor necrosis factor-alpha receptor-associated factor -6).

\section{Acknowledgements}

This study was financially supported by Kuwait Foundation for the Advancement of Sciences (KFAS). We thank Rawan Edan for her support in real time PCR. We thankfully acknowledge the technical help by Mr. Azadali K. Moorji and the staff of the Tissue Bank Core Facility.

\section{Disclosure Statement}

The authors declare that they have no competing interests.

\section{References}

1 Murphy G: Matrix metalloproteinases and their inhibitors. Acta Orthop Scand Suppl 1995;266:55-60.

72 Visse R, Nagase H: Matrix metalloproteinases and tissue inhibitors of metalloproteinases: Structure, function, and biochemistry. Circ Res 2003;92:827-839.

3 Chen Y, Wei X, Guo C, Jin H, Han Z, Han Y, Qiao T, Wu K, Fan D: Runx3 suppresses gastric cancer metastasis through inactivation of mmp9 by upregulation of timp-1. Int J Cancer 2011;129:1586-1598.

4 Tian TV, Tomavo N, Huot L, Flourens A, Bonnelye E, Flajollet S, Hot D, Leroy X, de Launoit Y, DuterqueCoquillaud M: Identification of novel tmprss2:Erg mechanisms in prostate cancer metastasis: Involvement of mmp9 and plxna2. Oncogene 2014;33:2204-2214.

5 Castano R, Miedinger D, Maghni K, Ghezzo H, Trudeau C, Castellanos L, Wattiez M, Vandenplas O, Malo JL: Matrix metalloproteinase-9 increases in the sputum from allergic occupational asthma patients after specific inhalation challenge. Int Arch Allergy Immunol 2013;160:161-164.

6 Devereux G, Steele S, Jagelman T, Fielding S, Muirhead R, Brady J, Grierson C, Brooker R, Winter J, Fardon T, McCormick J, Huang JT, Miller D: An observational study of matrix metalloproteinase (mmp)-9 in cystic fibrosis. J Cyst Fibros 2014

7 Goetzl EJ, Banda MJ, Leppert D: Matrix metalloproteinases in immunity. J Immunol 1996;156:1-4.

8 Gong Y, Hart E, Shchurin A, Hoover-Plow J: Inflammatory macrophage migration requires mmp-9 activation by plasminogen in mice. J Clin Invest 2008;118:3012-3024.

-9 Andrade VL, Petruceli E, Belo VA, Andrade-Fernandes CM, Caetano Russi CV, Bosco AA, Tanus-Santos JE, Sandrim VC: Evaluation of plasmatic mmp-8, mmp-9, timp-1 and mpo levels in obese and lean women. Clin Biochem 2012;45:412-415. 
Ahmad et al.: FSL-1 Induces MMP-9 in THP-1 Cells

10 Hofmann U, Beyersdorf N, Weirather J, Podolskaya A, Bauersachs J, Ertl G, Kerkau T, Frantz S: Activation of cd4+ t lymphocytes improves wound healing and survival after experimental myocardial infarction in mice. Circulation 2012;125:1652-1663.

11 Wong Y, Sethu C, Louafi F, Hossain P: Lipopolysaccharide regulation of toll-like receptor-4 and matrix metalloprotease-9 in human primary corneal fibroblasts. Invest Ophthalmol Vis Sci 2011;52:2796-2803.

12 Kariko K, Weissman D, Welsh FA: Inhibition of toll-like receptor and cytokine signaling--a unifying theme in ischemic tolerance. J Cereb Blood Flow Metab 2004;24:1288-1304.

$>13$ Wang JH, Kwon HJ, Jang YJ: Staphylococcus aureus increases cytokine and matrix metalloproteinase expression in nasal mucosae of patients with chronic rhinosinusitis and nasal polyps. Am J Rhinol Allergy 2010;24:422-427.

14 Maretti-Mira AC, de Pinho Rodrigues KM, de Oliveira-Neto MP, Pirmez C, Craft N: Mmp-9 activity is induced by leishmania braziliensis infection and correlates with mucosal leishmaniasis. Acta Trop 2011;119:160164.

15 Vanlaere I, Libert C: Matrix metalloproteinases as drug targets in infections caused by gram-negative bacteria and in septic shock. Clin Microbiol Rev 2009;22:224-239, Table of Contents.

16 Wang YY, Myhre AE, Pettersen SJ, Dahle MK, Foster SJ, Thiemermann C, Bjornland K, Aasen AO, Wang JE: Peptidoglycan of staphylococcus aureus induces enhanced levels of matrix metalloproteinase-9 in human blood originating from neutrophils. Shock 2005;24:214-218.

17 Lu Y, Wahl LM: Production of matrix metalloproteinase-9 by activated human monocytes involves a phosphatidylinositol-3 kinase/akt/ikkalpha/nf-kappab pathway. J Leukoc Biol 2005;78:259-265.

18 Shibata K, Hasebe A, Into T, Yamada M, Watanabe T: The n-terminal lipopeptide of a 44-kda membranebound lipoprotein of mycoplasma salivarium is responsible for the expression of intercellular adhesion molecule-1 on the cell surface of normal human gingival fibroblasts. J Immunol 2000;165:6538-6544.

19 Okusawa T, Fujita M, Nakamura J, Into T, Yasuda M, Yoshimura A, Hara Y, Hasebe A, Golenbock DT, Morita M, Kuroki Y, Ogawa T, Shibata K: Relationship between structures and biological activities of mycoplasmal diacylated lipopeptides and their recognition by toll-like receptors 2 and 6. Infect Immun 2004;72:16571665.

$\longrightarrow 20$ Livak KJ, Schmittgen TD: Analysis of relative gene expression data using real-time quantitative pcr and the 2(-delta delta $\mathrm{c}(\mathrm{t})$ ) method. Methods 2001;25:402-408.

21 Rose WA, 2nd, McGowin CL, Pyles RB: Fsl-1, a bacterial-derived toll-like receptor 2/6 agonist, enhances resistance to experimental hsv-2 infection. Virol J 2009;6:195.

22 Sato H, Seiki M: Regulatory mechanism of $92 \mathrm{kda}$ type iv collagenase gene expression which is associated with invasiveness of tumor cells. Oncogene 1993;8:395-405.

23 Rhee JW, Lee KW, Kim D, Lee Y, Jeon OH, Kwon HJ, Kim DS: Nf-kappab-dependent regulation of matrix metalloproteinase-9 gene expression by lipopolysaccharide in a macrophage cell line raw 264.7. J Biochem Mol Biol 2007;40:88-94.

24 Shamsul HM, Hasebe A, Iyori M, Ohtani M, Kiura K, Zhang D, Totsuka Y, Shibata K: The toll-like receptor 2 (tlr2) ligand fsl-1 is internalized via the clathrin-dependent endocytic pathway triggered by cd14 and cd36 but not by tlr2. Immunology 2010;130:262-272.

25 Triantafilou M, Manukyan M, Mackie A, Morath S, Hartung T, Heine H, Triantafilou K: Lipoteichoic acid and toll-like receptor 2 internalization and targeting to the golgi are lipid raft-dependent. J Biol Chem 2004;279:40882-40889.

-26 Brandt KJ, Fickentscher C, Kruithof EK, de Moerloose P: Tlr2 ligands induce nf-kappab activation from endosomal compartments of human monocytes. PLoS One 2013;8:e80743.

27 Akira S, Takeda K: Toll-like receptor signalling. Nat Rev Immunol 2004;4:499-511.

-28 Bond M, Fabunmi RP, Baker AH, Newby AC: Synergistic upregulation of metalloproteinase-9 by growth factors and inflammatory cytokines: An absolute requirement for transcription factor nf-kappa b. FEBS Lett 1998;435:29-34.

29 Himelstein BP, Lee EJ, Sato H, Seiki M, Muschel RJ: Transcriptional activation of the matrix metalloproteinase-9 gene in an h-ras and v-myc transformed rat embryo cell line. Oncogene 1997;14:1995-1998.

-30 Maeda A, Sobel RA: Matrix metalloproteinases in the normal human central nervous system, microglial nodules, and multiple sclerosis lesions. J Neuropathol Exp Neurol 1996;55:300-309. 


\begin{tabular}{|c|c|c|}
\hline Cellular P & Cell Physiol Biochem 2014;34:929-942 & \\
\hline and Biochemistry & $\begin{array}{l}\text { DOI: 10.1159/000366310 } \\
\text { Published online: August 26, } 2014\end{array}$ & $\begin{array}{l}\text { O 2014 S. Karger AG, Basel } \\
\text { www.karger.com/cpb }\end{array}$ \\
\hline
\end{tabular}

31 Mignatti P, Rifkin DB: Plasminogen activators and matrix metalloproteinases in angiogenesis. Enzyme Protein 1996;49:117-137.

-32 Aliprantis AO, Weiss DS, Radolf JD, Zychlinsky A: Release of toll-like receptor-2-activating bacterial lipoproteins in shigella flexneri culture supernatants. Infect Immun 2001;69:6248-6255.

-33 Medzhitov R, Janeway CA, Jr.: Innate immunity: The virtues of a nonclonal system of recognition. Cell 1997;91:295-298.

-34 Schwandner R, Dziarski R, Wesche H, Rothe M, Kirschning CJ: Peptidoglycan- and lipoteichoic acid-induced cell activation is mediated by toll-like receptor 2. J Biol Chem 1999;274:17406-17409.

35 Takeda K, Kaisho T, Akira S: Toll-like receptors. Annu Rev Immunol 2003;21:335-376.

-36 Takeuchi O, Hoshino K, Kawai T, Sanjo H, Takada H, Ogawa T, Takeda K, Akira S: Differential roles of tlr2 and tlr4 in recognition of gram-negative and gram-positive bacterial cell wall components. Immunity 1999;11:443-451.

-37 Yoshimura A, Lien E, Ingalls RR, Tuomanen E, Dziarski R, Golenbock D: Cutting edge: Recognition of grampositive bacterial cell wall components by the innate immune system occurs via toll-like receptor 2. J Immunol 1999;163:1-5.

38 Beutler B, Du X, Poltorak A: Identification of toll-like receptor 4 (tlr4) as the sole conduit for lps signal transduction: Genetic and evolutionary studies. J Endotoxin Res 2001;7:277-280.

39 Kalis C, Kanzler B, Lembo A, Poltorak A, Galanos C, Freudenberg MA: Toll-like receptor 4 expression levels determine the degree of lps-susceptibility in mice. Eur J Immunol 2003;33:798-805.

40 Lavelle EC, Murphy C, O'Neill LA, Creagh EM: The role of tlrs, nlrs, and rlrs in mucosal innate immunity and homeostasis. Mucosal Immunol 2010;3:17-28.

41 Kawai T, Adachi 0, Ogawa T, Takeda K, Akira S: Unresponsiveness of myd88-deficient mice to endotoxin. Immunity 1999;11:115-122.

42 Takeuchi 0, Hoshino K, Akira S: Cutting edge: Tlr2-deficient and myd88-deficient mice are highly susceptible to staphylococcus aureus infection. J Immunol 2000;165:5392-5396.

43 Ahmad R, El Bassam S, Cordeiro P, Menezes J: Requirement of tlr2-mediated signaling for the induction of il-15 gene expression in human monocytic cells by hsv-1. Blood 2008;112:2360-2368.

$\checkmark 44$ Takeuchi O, Takeda K, Hoshino K, Adachi O, Ogawa T, Akira S: Cellular responses to bacterial cell wall components are mediated through myd88-dependent signaling cascades. Int Immunol 2000;12:113-117.

45 Newton K, Dixit VM: Signaling in innate immunity and inflammation. Cold Spring Harb Perspect Biol 2012;4

46 Bauerfeld CP, Rastogi R, Pirockinaite G, Lee I, Huttemann M, Monks B, Birnbaum MJ, Franchi L, Nunez G, Samavati L: Tlr4-mediated akt activation is myd88/trif dependent and critical for induction of oxidative phosphorylation and mitochondrial transcription factor a in murine macrophages. J Immunol 2012;188:2847-2857.

47 Adachi O, Kawai T, Takeda K, Matsumoto M, Tsutsui H, Sakagami M, Nakanishi K, Akira S: Targeted disruption of the myd88 gene results in loss of il-1- and il-18-mediated function. Immunity 1998;9:143150.

48 Gracie JA, Robertson SE, McInnes IB: Interleukin-18. J Leukoc Biol 2003;73:213-224.

-49 Eaves-Pyles T, Szabo C, Salzman AL: Bacterial invasion is not required for activation of nf-kappab in enterocytes. Infect Immun 1999;67:800-804.

-50 Hauf N, Goebel W, Fiedler F, Sokolovic Z, Kuhn M: Listeria monocytogenes infection of p388d1 macrophages results in a biphasic nf-kappab (rela/p50) activation induced by lipoteichoic acid and bacterial phospholipases and mediated by ikappabalpha and ikappabbeta degradation. Proc Natl Acad Sci U S A 1997;94:9394-9399.

51 Gupta D, Wang Q, Vinson C, Dziarski R: Bacterial peptidoglycan induces cd14-dependent activation of transcription factors creb/atf and ap-1. J Biol Chem 1999;274:14012-14020.

52 Rawadi G, Ramez V, Lemercier B, Roman-Roman S: Activation of mitogen-activated protein kinase pathways by mycoplasma fermentans membrane lipoproteins in murine macrophages: Involvement in cytokine synthesis. J Immunol 1998;160:1330-1339.

-53 Wilhelmsen K, Mesa KR, Lucero J, Xu F, Hellman J: Erk5 protein promotes, whereas mek1 protein differentially regulates, the toll-like receptor 2 protein-dependent activation of human endothelial cells and monocytes. J Biol Chem 2012;287:26478-26494. 
54 Baseman JB, Tully JG: Mycoplasmas: Sophisticated, reemerging, and burdened by their notoriety. Emerg Infect Dis 1997;3:21-32.

55 Taylor-Robinson D, Schaeverbeke T: Mycoplasmas in rheumatoid arthritis and other human arthritides. J Clin Pathol 1996;49:781-782.

56 Blanchard A, Montagnier L: Aids-associated mycoplasmas. Annu Rev Microbiol 1994;48:687-712.

57 Hazzalin CA, Cano E, Cuenda A, Barratt MJ, Cohen P, Mahadevan LC: P38/rk is essential for stress-induced nuclear responses: Jnk/sapks and c-jun/atf-2 phosphorylation are insufficient. Curr Biol 1996;6:10281031.

58 Cano E, Hazzalin CA, Kardalinou E, Buckle RS, Mahadevan LC: Neither erk nor jnk/sapk map kinase subtypes are essential for histone h3/hmg-14 phosphorylation or c-fos and c-jun induction. J Cell Sci 1995;108:3599-3609.

59 Cano E, Doza YN, Ben-Levy R, Cohen P, Mahadevan LC: Identification of anisomycin-activated kinases p45 and p55 in murine cells as mapkap kinase-2. Oncogene 1996;12:805-812.

60 Gordon GM, Ledee DR, Feuer WJ, Fini ME: Cytokines and signaling pathways regulating matrix metalloproteinase-9 (mmp-9) expression in corneal epithelial cells. J Cell Physiol 2009;221:402-411.

61 Karin M: The regulation of ap-1 activity by mitogen-activated protein kinases. J Biol Chem 1995;270:16483-16486.

62 Papa S, Zazzeroni F, Pham CG, Bubici C, Franzoso G: Linking jnk signaling to nf-kappab: A key to survival. J Cell Sci 2004;117:5197-5208. 